\title{
Colgajo de piel en isla con pedículo fasciocutáneo para cuello: caso clínico
}

\section{Island skin flap with fasciocutaneous pedicle for neck reconstruction: case report}

\author{
Ramón Vera, J .L.*, Olaya Martínez, H.E.**, Trespalacios González, A.E.***, \\ Álvarez Díaz, M.A.*'*k*
}

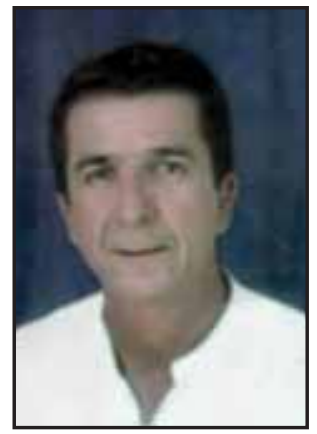

Ramón Vera, J .L.

\section{Resumen}

Los defectos por falta de piel o las secuelas de quemaduras a nivel del cuello presentan gran dificultad de cobertura debido a la escasez de tejidos locales útiles para corregirlos, ya que debemos dar una solución a las graves alteraciones funcionales y estéticas que producen, con un mínimo de secuelas.

Presentamos un caso clínico con graves secuelas de quemaduras en cuello que producían retracción importante y que fue resuelto con un colgajo de piel en isla con pedículo fasciosubcutáneo tomado de región subclavicular. Este colgajo presenta irrigación cutánea por medio de arterias cutáneas directas provenientes de la arteria mamaria interna y brinda la oportunidad de solucionar alteraciones a nivel del cuello con gran facilidad para su realización y con mínimas secuelas en la zona donante.

\author{
Palabras clave Reconstrucción cuello, Colgajo fascio- \\ cutáneo, Colgajo en isla \\ Código numérico 24-242-2423
}

\begin{abstract}
Cutaneous defects or burn sequels at the neck level present difficult of covering, due to the poor local tissues, although we should give a solution to the serious functional and aesthetic alterations with minimal sequels.

We present a clinical case with burn sequels of the neck that produced important retraction and that was solved whit an island fasciocutaneous pedicle flap from subclavicular region.

This flap has skin irrigation supported by the cutaneous arteries coming from the internal mammary artery and gives us the opportunity to solve these alterations at the neck level in an easy way and with minimal sequels in the donor area.
\end{abstract}

$\begin{array}{ll}\text { Key words } & \begin{array}{l}\text { Neck reconstruction, Fasciocutaneous } \\ \text { flap, Island flap }\end{array} \\ \text { Numeral Gode } & 24-242-2423\end{array}$

\footnotetext{
* Profesor de Cirugía Plástica, Universidad Surcolombiana. 


\section{Introducción}

Para la cobertura de las pérdidas de sustancia a nivel del cuello, ya sean producidas por resección oncológica o por quemaduras, debemos procurar una reparación que nos dé protección a los elementos nobles del cuello y que también nos permita restituir la función y estética del mismo.

Las técnicas de reconstrucción a base de injertos de piel presentan pésimos resultados por su gran retracción, existiendo acuerdo general sobre la superioridad de las reconstrucciones que emplean tejidos vascularizados.

Un procedimiento de indudable interés, preconizado en Argentina por Correa Iturraspe, es el colgajo abierto cervicoacromial, también denominado "colgajo en charretera" (1); tiene como inconveniente principal la lenta cicatrización de la zona donante y el que en la mayoría de los casos requiere un injerto de piel adicional; otro problema importante es su escaso arco de rotación, lo que dificulta la reconstrucción de zonas cervicales laterales; finalmente su grosor, que puede ser antiestético, especialmente en pacientes obesos (2).

Otro colgajo de gran utilidad en la reconstrucción del cuello es el colgajo miocutaneo de pectoral mayor en isla, sin lugar a dudas el más utilizado en Cirugía Reconstructiva de cuello. Se han subrayado algunos inconvenientes de este colgajo, como su excesivo espesor, sobre todo en pacientes con mucho panículo adiposo y también la cicatriz antiestética que deja en la zona donante, especialmente en mujeres (3-6).

\section{CASO CLINICO}

Presentamos el caso de una mujer de 46 años de edad, intervenida en dos ocasiones por secuelas de quemadura en el cuello producida con gasolina (injertos de piel parcial y posteriormente cirugía mediante zetaplastias); presentaba persistencia de brida cérvico- torácica. Se propuso reconstrucción mediante un colgajo de piel en isla con pedículo fasciocutaneo (Fig. 1).

Con la paciente en decúbito dorsal y en hiperextension de cuello, se realiza degollado con bisturí y disección con electrocauterio hasta lograr la extensión completa del cuello, quedando una zona denudada en forma de uso de aproximadamente $12 \times 5 \mathrm{~cm}$ de diámetro.

Se realiza la marcación de piel con esta forma y medida a nivel subclavicular, con el eje mayor paralelo a la misma (Fig. 2). Incidimos con el bisturí hasta la fascia superficial, que incluimos, y comenzamos a disecar un colgajo fasciocutáneo de aproximadamen-

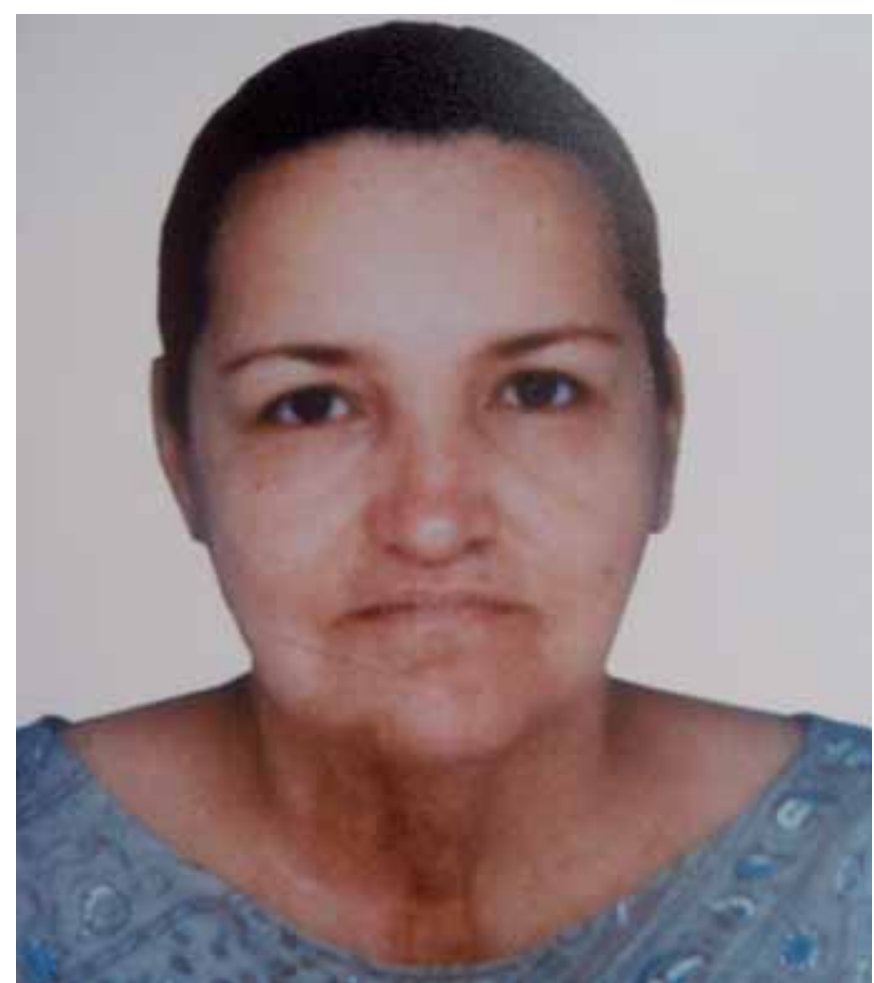

Fig. 1. Mujer de 46 años de edad que presenta cicatriz retráctil en cuello, secuela de quemadura.

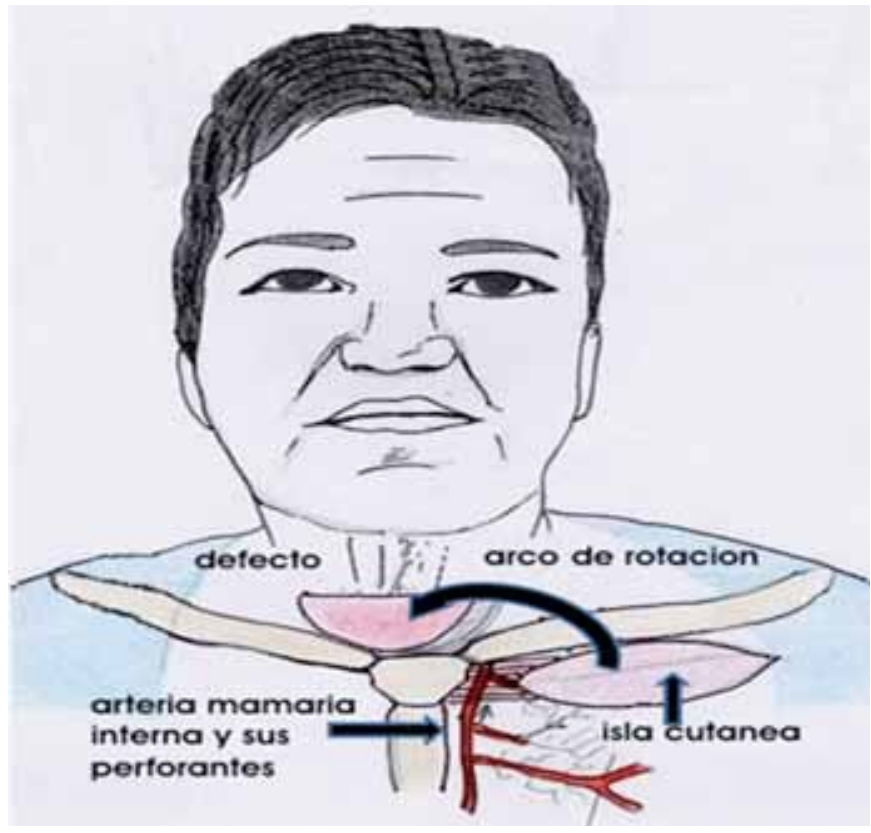

Fig. 2. Diseño de colgajo fasciosubcutáneo en región infraclavicular.

transverso irrigado por las perforantes de la arteria mamaria interna hacia la línea media esternal, donde con un arco de rotación de $90^{\circ}$, podemos deslizarlo por un túnel dermoepidérmico previamente realizado hacia la parte superior del cuello; aquí, ya en el defecto, suturamos el plano dérmico con monofilamento reabsorbible 4.0 y la piel con monofilamento no absorbible 4.0. Finalmente cubrimos con Micropore ${ }^{\circledR}$ y apósitos con escasa compresión; en la zona donante se realiza despegamiento y cierre directo por planos del defecto creado. 


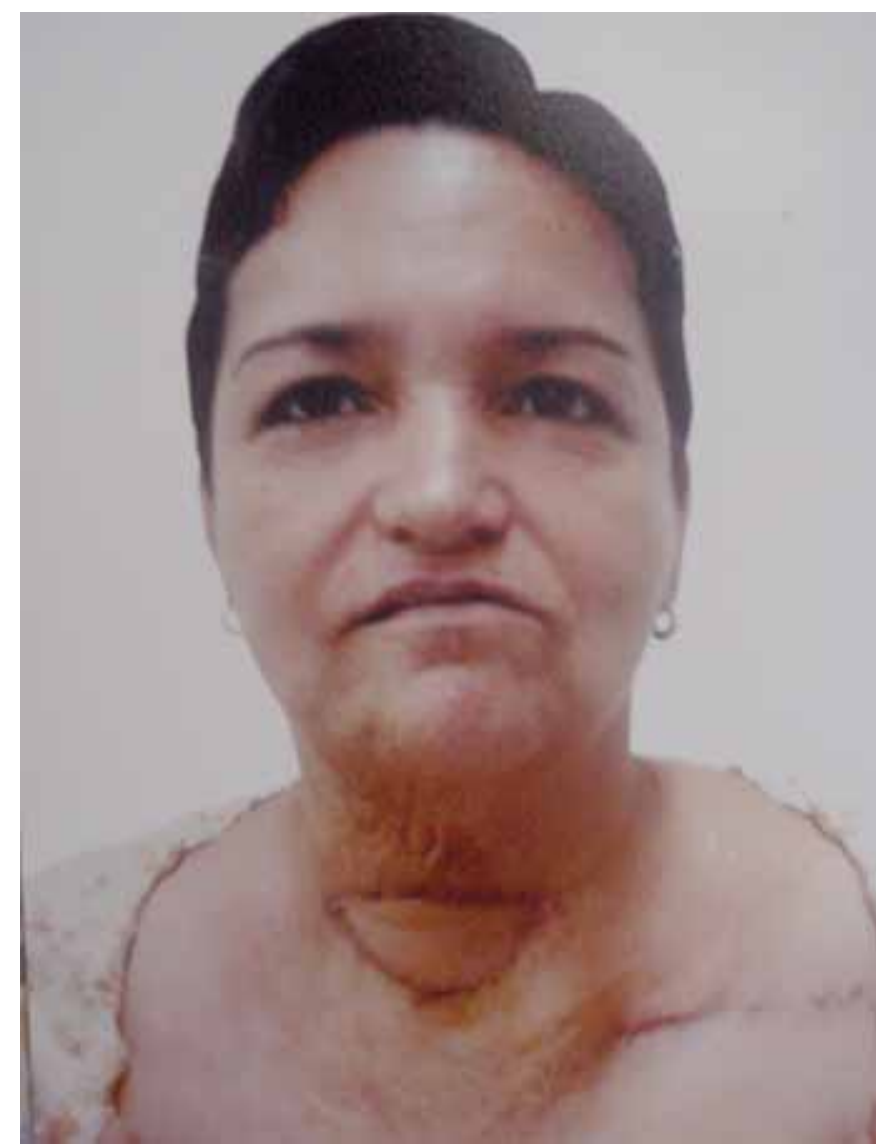

Fig. 3. Postoperatorio al mes de la intervención: reconstrucción mediante colgajo de piel en isla con pedículo fasciocutáneo.

Resultados

Con la técnica descrita, logramos en la paciente el objetivo reconstructivo funcional con movimientos de flexo-extensión y arcos de rotación completos, permanentes y sin ninguna complicación. Fue dada de alta hospitalaria a las 24 horas de la cirugía y presentaba una resolución muy adecuada de las cicatrices al mes de la intervención (Fig. 3).

\section{Discusión}

Las secuelas más frecuentes de las quemaduras en el cuello son las cicatrices hipertróficas y las bridas cicatriciales, que provocan pérdida del ángulo mandibular, pérdida de movilidad del cuello y la dificultad añadida que supone la intubación de estos pacientes, que normalmente son subsidiarios de múltiples intervenciones reconstructivas.

Existen diversas posibilidades para reconstruir estos importantes defectos del cuello, como son los injertos de piel con o sin zeta-plastias, que en la mayoría de veces conllevan recidivas incluso peores dado el comportamiento de la piel injertada. Logramos mejores resultados con la escisión del defecto seguida de aporte cutáneo mediante colgajos locales, a distancia o microquirúrgicos o con el uso de expansores.
Para recubrir los defectos superficiales del cuello, deberían utilizarse siempre colgajos de escaso espesor y de un color y textura similares a los de la piel del cuello; los tejidos de características más similares los aportan los colgajos locales y regionales como son los colgajos miocutáneos de pectoral mayor y de deltopectoral, que siguen siendo de gran utilidad a pesar de que, como secuelas inestéticas, requieran injerto en la zona donante y produzcan cicatrices abultadas.

Frente a todo esto, las ventajas de la opción que presentamos empleando el colgajo de piel en isla con pedículo fasciocutáneo, se basan en la simplicidad de la técnica y en que se realiza sobre un mismo campo operatorio, con escasas o nulas secuelas funcionales ni estéticas, en que no hay necesidad de procedimientos adicionales para adelgazamiento de la cicatriz y finalmente, en que se pueden diseñar colgajos bilaterales de acuerdo a las necesidades del defecto.

Los colgajos fasciocutáneos son de relativa reciente aparición dentro de la Cirugía Plástica; se desarrollaron a principios de la década de los noventa respondiendo a un entendimiento más preciso de la anatomía de la piel y de las fascias superficial y profunda, constituyendo un punto de evolución más de los colgajos fasciocutáneos descritos por Poten en 1981 (6).

Posteriormente los estudios de Nakajima y Minabe (7), en la Universidad de Keio, Japón, definieron microanatómicamente la piel y la fascia, desarrollando una nueva clasificación fasciocutánea y permitiendo el desarrollo racionalizado de este nuevo tipo de colgajos; los trabajos de Batchelor y Rahim (8) analizan la red vascular mediante examen microanatómico en un sentido tridimensional y determinan que el sistema fasciocutáneo consiste en una red de arterias perforantes que emergen a la superficie a lo largo de septos fasciales entre vientres musculares adyacentes y que luego se ramifican a nivel de la fascia profunda para formar al menos tres plexos vasculares descritos, denominados subfascial, intrafascial, y suprafascial, del cual se emiten ramas que van a irrigar el tejido celular subcutáneo y la dermis.

Los trabajos de Haertsch, en 1981 (9) demostraron además que el plexo localizado en la parte superficial de la fascia profunda suprafascial, es el más importante, pues el calibre de sus vasos es mayor, así como el número de anastomosis existentes, mientras que aquellos vasos situados por debajo de la fascia, plexo subfascial, vasos tipo II y III, son más pequeños y más delicados que los suprafasciales.

Tanto Lang como Schäfer (10) han demostrado que los plexos fasciales están conectados por un número variable de vasos interarteriolares y anastomóticos intervenulares. Los plexos subfascial y suprafascial discurren entre el tejido areolar laxo que contiene 
islas de células adiposas, mientras que los vasos del plexo intrafascial lo hacen entre los haces de tejido colágeno.

Como podemos observar por todos estos datos, la fascia profunda y la superficial tienen una irrigación propia que se debe a que estas estructuras son dos remanentes de dos sistemas embriológicamente distintos pero poco desarrollados en los seres humanos y con mejor desarrollo en los simios. Tales estructuras son el panículo "carnosum" que da origen a la fascia profunda y el panículo "adiposum" que da origen a la fascia superficial. Cada una al ser una estructura embriológicamente diferente, tiene un sistema vascular propio, distinguible al de la piel, lo cual evidentemente es clave para el desarrollo teórico y práctico de los colgajos fasciocutáneos. Una de las ventajas de estos colgajos, como lo aseguró Gumener (11), es que su realización disminuye la cantidad de injertos de piel de espesor parcial requeridos para cubrir el defecto del área donante cuando se rota un colgajo fasciocutáneo clásico y también disminuyen la alteración estética resultante por pérdida de contorno.

\section{Conclusiones}

Creemos importante tener en cuenta el colgajo de piel en isla con pedículo fasciocutáneo para cubrir defectos del cuello dentro del escaso abanico de posibilidades de esta zona, ya que nos da una buena cobertura, es una técnica fácil, se realiza en un mismo campo operatorio y presenta excelentes resultados funcionales y estéticos.

\section{Dirección del autor}

\author{
Dr. José Lizardo Ramón Vera
}

Calle 25 no 1g-89, Reinaldo Matiz, Neiva, Huila, zona postal 1, Colombia.

e-mail: jolira01@hotmail.com

\section{Bibliografía}

1. Correa Iturraspe M, Fernandez J. C.: "Epaulet flaps. Their value in the repair of extensive losses of substance of the neck". Bol Trab Soc Sir B. Aires. 1959, 5;43:485.

2. Kirschbaum, S.M.: "Tratamiento de las quemaduras". En: Quemaduras y Cirugía Plástica de sus secuelas. Salvat Editores, S.A. Mallorca, 1979. Pp. 267-318

3. Jurado Ramos, A.: "Deltopectoral”. En: Fundamentos prácticos en cirugía reconstructiva cervicofacial. Junta de Andalucía, Consejería de Salud, 1990. Pp. 81-87.

4. Conley JJ.: "One-stage radical resection of cervical esophagus, larynx, pharynx, and neck, with immediate reconstruction”. AMA Arch Otolaryngol. 1953, 58(6):645.

5. Hueston JT, McConchie IH.: "A compound pectoral flap". Aust N Z J Surg. 1968, 38(1):61.

6. Poten B.: "The fasciocutaneous flap: its use in soft tissue defects of the lower leg". Br. J. Plast. Surg. 1981, 34: 215.

7. Nakajima H., Minabe T, Imanishi N.: "Three dimensional analysis and classification of arteries in the skin and subcutaneous adipofascial tissue by computer graphics imaging". Plast. Reconst Surg. 1998, 102:748.

8. Batchelor JS, Rahim A, McGuinness A.: "The anatomic basis for arteriovenous shunting in human lower leg fascial flaps". Plast Reconstr Surg. 1995, 95(2):233; discussion 240.

9. Haertsch PA.: "The blood supply to the skin of the leg: a post-mortem investigation". Br J Plast Surg. 1981, 34(4):470.

10. Cormarck GC, Lamberty GH.: "The microcirculation". In: The arterial anatomy of the skin flaps. 2a ed. New York: Churchill Livingstone

11. Gumener R, Zbrodowski A, Montandon D.: "The reversed fasciosubcutaneous flap in the leg". Plast Reconstr Surg. 1991, 88(6): 1034; discussion 1042. 